\title{
Salt Effects on the Phase Behavior, Structure, and Rheology of Chromonic Liquid Crystals
}

\author{
Andrei F. Kostko,,$+\star$ Bani H. Cipriano, \\ Mikhail A. Anisimov, ${ }^{*}$ Dganit Danino, ${ }^{\S}$ and Srinivasa R. Raghavan*,* \\ Department of Chemical Engineering, University of Maryland, College Park, Maryland 20742-2111, and \\ Department of Biotechnology and Food Engineering, Technion-Israel Institute of Technology, \\ Technion, Haifa, Israel, 32000
}

Received: May 16, 2005; In Final Form: August 17, 2005

\begin{abstract}
Chromonic liquid crystals are formed by the addition of aromatic molecules such as disodium chromoglycate (cromolyn) to water. In this study, we investigate the addition of salts to the lyotropic nematic phase of cromolyn aqueous solutions. The addition of sodium and potassium salts shifts the isotropic-nematic phase boundary upward by more than $10^{\circ} \mathrm{C}$, so that samples that were isotropic at room temperature are transformed into nematic phases. Salt effects are predominantly dictated by the cation, not the anion, and appear to differ based on cation size. In contrast to small, hydrated cations like sodium, large, weakly hydrated cations such as tetraethylammonium and tetrabutylammonium shift the phase boundary downward, thus stabilizing the isotropic phase at the expense of the nematic one. The phase behavior results are highly correlated with viscosity measurements, with an upward shift in the phase boundary correlating with an increase in solution viscosity and vice versa. We also probe the microstructure in cromolyn-salt solutions, both indirectly by small-angle neutron scattering (SANS) and directly by cryo-transmission electron microscopy (cryo-TEM). The cryo-TEM images show the presence of rodlike aggregates that possibly undergo a higher order aggregation into bundles in the presence of salt.
\end{abstract}

\section{Introduction}

In recent years, the existence of a second class of aqueous lyotropic mesophases, termed chromonic liquid crystals (LCs), has come to be better recognized and understood. ${ }^{1-3}$ Unlike typical lyotropic phases formed by amphiphilic molecules having a hydrophilic head and a hydrophobic tail, chromonic LCs are formed by water-soluble molecules that contain planar aromatic rings. Examples of chromonic LC-forming molecules include drugs, dyes, and nucleic acids. ${ }^{4,5}$ Among these, the most widely studied has been disodium chromoglycate, or cromolyn, which is sold as an anti-asthmatic drug under the trade name Intal (there is no apparent link between its therapeutic effect and its chromonic nature) ${ }^{6-10}$ The structure of cromolyn is presented in Figure 1.

Chromonic LCs are still not understood to the same extent as amphiphile-based lyotropic LCs. Lydon has recently summarized the current state of knowledge on chromonics in two excellent reviews. ${ }^{2,3}$ Note that chromonic molecules do not show a clear separation of hydrophilic and hydrophobic parts since the hydrophilic (ionic or hydrogen-bonding) groups that impart water solubility are distributed all around the periphery of the hydrophobic aromatic rings. Consequently, chromonic molecules do not form micelles, nor do they show any appreciable surface activity. However, the presence of planar aromatic $\pi$-rings on these molecules leads to an attractive $\pi-\pi$ interaction, resulting in a face-to-face stacking of the rings. This leads to columnar

\footnotetext{
* Address correspondence to this author. E-mail: sraghava@eng.umd.edu

$\dagger$ Present address: Department of Chemical Engineering, Virginia Commonwealth University, Richmond, VA; also affiliated with St. Petersburg State University of Refrigeration and Food Engineering, St. Petersburg, 191002, Russia.

$\doteqdot$ University of Maryland.

$\S$ Technion-Israel Institute of Technology.
}

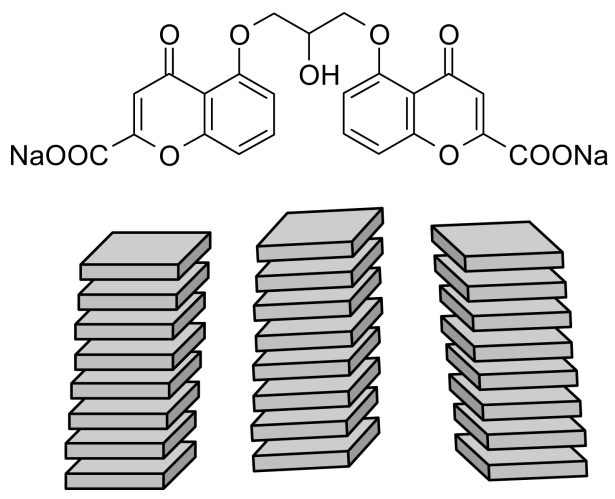

Figure 1. Chemical structure of cromolyn and postulated microstructure of cromolyn aggregates in the nematic $(\mathrm{N})$ phase. The aggregates are visualized to be columns formed by the stacking of cromolyn molecules. In the nematic phase, the aggregates show orientational, but not positional, order.

aggregates of chromonic molecules, and these are formed in isotropic solutions as well. ${ }^{11}$ The nematic $\mathrm{N}$ phase corresponds to a uniaxial stacking of these columns with no positional order (Figure 1). At higher concentrations, a hexagonal $\mathrm{M}$ phase also occurs, with both orientational and positional order of the columns.

Important questions remain about the driving force for chromonic LC formation and for the columnar aggregation that precedes it. The crucial role of $\pi-\pi$ interactions suggests that the aggregation is enthalpy-driven, unlike the entropy-driven hydrophobic effect that controls the aggregation of amphiphiles into micelles. ${ }^{2}$ If so, what role, if any, does the solvent (water) play? What about the ionic interactions between adjacent chromonic aggregates? How does the addition of salt or other additives influence the aggregation? These are some of the 
unanswered questions that provide the motivation for this study. The importance of these questions extends also to organic and biochemistry, since the stacking of dyes and nucleic acid bases is controlled by a similar mechanism.

So far, only a few studies have investigated the effect of salt or other additives on chromonic nematic LCs. ${ }^{12-16}$ The addition of alkali metal chlorides $(\mathrm{NaCl}, \mathrm{KCl})$ to cromolyn aqueous solutions was reported to increase the isotropic-nematic transition temperature by several degrees. ${ }^{12-14}$ Salt effects in these studies were restricted to a single concentration of cromolyn and a limited range of salt concentrations. Systematic studies with different salts have not been reported to our knowledge. Interestingly, salt has a much smaller effect on the transition temperatures of micellar nematics formed by ionic surfactants. ${ }^{17}$ In both micellar and chromonic nematics, salt effects have been interpreted in terms of changes in electrostatic interactions. For example, the screening of ionic interactions between cromolyn molecules by added salt was speculated to induce "stiffer and longer aggregates" of cromolyn. ${ }^{13}$ However, no structural evidence for such aggregate growth has been reported, to our knowledge.

In the present study, we systematically explore the addition of salt to cromolyn aqueous solutions using a combination of phase behavior observations, rheology, small-angle neutron scattering (SANS), and cryo-transmission electron microscopy (cryo-TEM). We study a number of salts as well as other chemicals that are known to perturb the structure of water. Our findings confirm that salts can drastically alter the phase behavior of cromolyn: by varying the salt species, we can shift the isotropic-nematic phase boundaries upward or downward by several degrees in temperature. The origin of these effects, however, seems to lie not in electrostatic interactions per se, but in how the salt cations participate in aggregate formation. In addition, salts also drastically increase the viscosity of the nematic cromolyn solutions, suggesting that the columnar aggregates are longer in the presence of salt. Our study thus underscores the role of salt cations in dictating the structure and properties of chromonic mesophases.

\section{Experimental Section}

Materials. Cromolyn of purity ca. 95\% was obtained from Sigma, as were the following salts: tetraethylammonium bromide and tetrabutylammonium bromide. Sodium sulfate, sodium chloride, ammonium sulfate, and urea were obtained from J. T. Baker. Sodium citrate (2-hydroxy-1,2,3-propanetricarboxylic acid, trisodium salt), sodium perchlorate, sodium bromide, sodium acetate, sodium nitrate, potassium chloride, lithium chloride, ammonium chloride, and glycerol were purchased from Fisher Scientific. Finally, ethylene glycol and methanol were purchased from Aldrich. All salts and chemicals were $>99 \%$ in purity and were used as received without further purification.

Sample Preparation. Deionized water was used in preparing all the solutions except those for SANS, for which $\mathrm{D}_{2} \mathrm{O}$ (Cambridge Isotopes) was used. Solutions were prepared by mixing the appropriate components in glass vials, followed by mild heat while stirring. The homogeneous solutions were then cooled to room temperature and stored overnight before further experimentation.

Phase Behavior. Phase behavior was recorded by visual observation. Sample vials were placed in a thermostated water bath and were observed directly, as well as under crossed polarizers, at different temperatures. The phase boundaries as a function of temperature were determined always while traversing

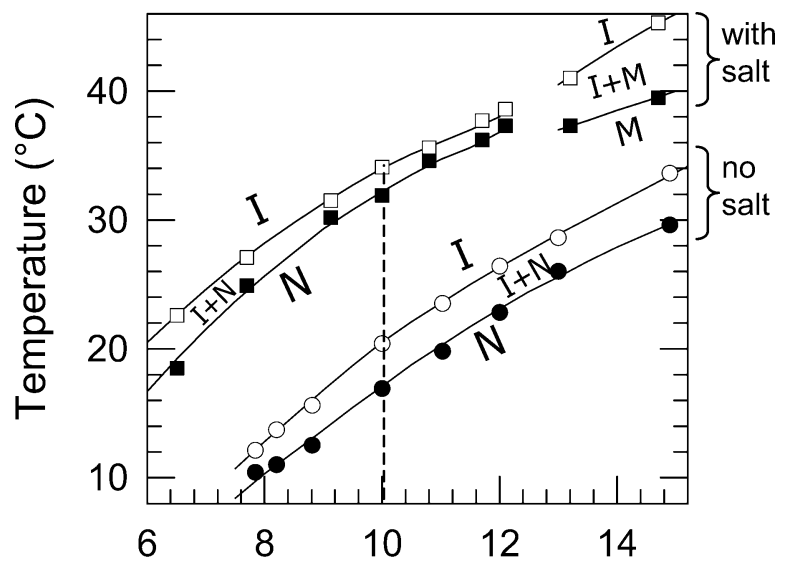

\section{wt. \% cromolyn}

Figure 2. Partial phase diagram for cromolyn aqueous solutions with and without added salt $(310 \mathrm{mM} \mathrm{NaCl})$. The label I refers to the isotropic phase, $\mathrm{N}$ the nematic phase, and $\mathrm{M}$ the middle or hexagonal phase. $\mathrm{I}+\mathrm{N}$ and $\mathrm{I}+\mathrm{M}$ denote the 2-phase regions. Note that a composition of $10 \mathrm{wt} \%$ cromolyn, shown by the dashed line, is isotropic at room temperature $\left(25^{\circ} \mathrm{C}\right)$, but becomes nematic upon addition of salt.

from a 1-phase to a 2-phase region. The transition temperature corresponds to the incipient formation of a second phase in an initially homogeneous sample. Incremental temperature steps of $0.2^{\circ} \mathrm{C}$ were taken near the transition temperature to accurately locate the point. The phase transition temperatures reported here are reproducible to $\pm 0.5^{\circ} \mathrm{C}$.

Rheology. Rheological experiments were performed on an AR2000 stress-controlled rheometer (TA Instruments). A coneand-plate geometry (cone diameter, $40 \mathrm{~mm}$ and cone angle, 0.04 rad) equipped with Peltier-based temperature control was used. A solvent trap was used to minimize sample evaporation.

SANS. SANS measurements were made on the NG-1 (8 m) beamline at NIST in Gaithersburg, MD. Samples were studied in $2 \mathrm{~mm}$ quartz cells. The scattering spectra were corrected and placed on an absolute scale, using calibration standards provided by NIST. The data are shown for the radially averaged, absolute intensity $I$ versus the scattering vector $q=(4 \pi / \lambda) \sin (\theta / 2)$, where $\lambda$ is the wavelength of incident neutrons and $\theta$ the scattering angle.

Cryo-TEM. The cryo-TEM work was done at the Hanna and Georg Krumholz Laboratory for Advanced Microsocpy at Technion-IIT. Samples for cryo-TEM were prepared in a controlled environment vitrification system (CEVS). In this apparatus, samples at the desired temperature are quenched rapidly into liquid ethane at its freezing point of $-183{ }^{\circ} \mathrm{C}$, forming a vitrified specimen, and then transferred to liquid nitrogen for storage. The vitrified samples were examined on a Philips CM120 microscope operated at $120 \mathrm{kV}$, using an Oxford CT-3500 cryo-holder for keeping the samples below $-180{ }^{\circ} \mathrm{C}$. Digital images were recorded in the minimal electron dose mode by a Gatan 791 MultiScan cooled CCD camera.

\section{Results}

Phase Behavior of Cromolyn-Salt Solutions. We first describe a partial phase diagram of cromolyn in water, both in the absence and in presence of salt (Figure 2). The salt of interest is $\mathrm{NaCl}$ and its concentration is fixed at $310 \mathrm{mM}$. The phase boundaries are plotted as a function of cromolyn concentration from 6 to $15 \mathrm{wt} \%$. Salt-free samples are observed to be nematic $(\mathrm{N})$ at low temperatures and, upon heating, undergo phase 


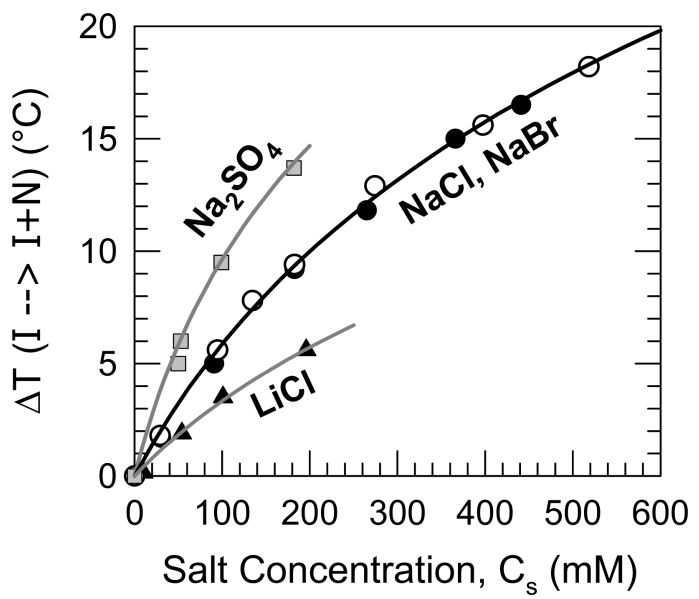

Figure 3. Shift in the $\mathrm{I} \rightarrow \mathrm{I}+\mathrm{N}$ phase transition temperature caused by the addition of salt to $10 \mathrm{wt} \%$ cromolyn solutions. Data are shown for the following salts as a function of salt concentration: $\mathrm{Na}_{2} \mathrm{SO}_{4}$ (squares), $\mathrm{NaCl}$ (filled circles), $\mathrm{NaBr}$ (open circles), and $\mathrm{LiCl}$ (triangles). The lines through the data are fits to eq 1 .

separation into two coexisting liquid phases, of which one is nematic and the other isotropic. Upon further heating, these twophase $(\mathrm{I}+\mathrm{N})$ samples turn isotropic (I). The phase behavior is thus defined by two coexistence curves, one for the $\mathrm{N} \rightarrow \mathrm{I}+\mathrm{N}$ transition (filled symbols) and the other for the $\mathrm{I} \rightarrow \mathrm{I}+\mathrm{N}$ transition (open symbols). In the presence of $310 \mathrm{mM} \mathrm{NaCl}$, both these coexistence curves are shifted upward by about 15 ${ }^{\circ} \mathrm{C}$, indicating that salt has a profound effect on the phase behavior. Taking a particular cromolyn concentration of $10 \mathrm{wt}$ $\%$ as an example (dashed line), the neat cromolyn sample is isotropic at room temperature $\left(25^{\circ} \mathrm{C}\right)$, but is transformed into a nematic phase upon addition of salt. At higher cromolyn concentrations, the $\mathrm{M}$ phase (middle, or hexagonal) emerges in lieu of the nematic phase and the corresponding two-phase region is a mixture of this phase and an isotropic solution (I+M). The $\mathrm{M}$ phase could be distinguished from the $\mathrm{N}$ phase based on their characteristic appearance under crossed polarizers.

To make a valid comparison of different salts, we decided to focus on the shift caused by salt on one of the coexistence curves, viz., the $\mathrm{I} \rightarrow \mathrm{I}+\mathrm{N}$ transition. We define the temperature shift $\Delta T(\mathrm{I} \rightarrow \mathrm{I}+\mathrm{N})$ as the difference between the phase transition temperature in the presence of salt and that for the neat cromolyn. We measured this $\Delta T$ for a fixed concentration of cromolyn, viz., $10 \mathrm{wt} \%$. Figure 3 reports $\Delta T$ as a function of salt concentration for three sodium salts and one lithium salt. We observe that $\Delta T$ increases monotonically with salt concentration for each salt. $\Delta T$ is largest for sodium sulfate $\left(\mathrm{Na}_{2} \mathrm{SO}_{4}\right)$, smaller and almost identical for sodium chloride and bromide ( $\mathrm{NaCl}$ and $\mathrm{NaBr}$ ), and the least for lithium chloride ( $\mathrm{LiCl}) . \mathrm{We}$ will see below that the $\Delta T$ values are higher for $\mathrm{Na}^{+}$ions than for all other cations (Figure 4). Also, we will see from Figure 5 that the higher $\Delta T$ for $\mathrm{Na}_{2} \mathrm{SO}_{4}$ arises simply because $2 \mathrm{~mol}$ of $\mathrm{Na}^{+}$is released for every mole of this salt in solution.

Since the effect of salts on the temperature shift $\Delta T$ is monotonic (Figure 3), we can compare different salts in terms of their $\Delta T$ at a fixed salt concentration. We chose a salt concentration of $100 \mathrm{mM}$ as the basis for comparison and the results for different salts are plotted as bar graphs in Figure 4. The shifts are seen to be practically identical for five monovalent sodium salts: sodium perchlorate $\left(\mathrm{NaClO}_{4}\right), \mathrm{NaBr}, \mathrm{NaCl}$, sodium acetate $\left(\mathrm{CH}_{3} \mathrm{COONa}\right)$, and sodium nitrate $\left(\mathrm{NaNO}_{3}\right)$. In other words, the anion has a negligible effect on the phase behavior. The higher $\Delta T$ for $\mathrm{Na}_{2} \mathrm{SO}_{4}$ (anion valency of two) and sodium citrate (anion valency of three) are simply due to the release of a higher concentration of $\mathrm{Na}^{+}$ions (see Figure 5 below). Experiments were also done keeping the same anion $\left(\mathrm{Cl}^{-}\right)$, while varying the cation in the salt. The $\Delta T$ for monovalent cations is found to follow the order $\mathrm{Na}^{+}>\mathrm{K}^{+}>$ $\mathrm{Li}^{+}>\mathrm{NH}_{4}{ }^{+}$. We conclude that sodium salts have the largest effect on cromolyn phase behavior, with potassium salts not far behind. Also, it is the salt cation that has the predominant effect, not the anion. Incidentally, we also studied chloride salts of divalent cations $\left(\mathrm{Mg}^{2+}\right.$ and $\left.\mathrm{Ca}^{2+}\right)$, but both of these gave a precipitate with cromolyn.

Figure 4 also shows that some additives shift the cromolyn phase behavior in the opposite direction-they tend to lower the coexistence curves, i.e., they stabilize the isotropic phase. Among the additives studied, ethylene glycol, glycerol, methanol, and urea all induced small, but negative $\Delta T$ values. Ethanol gave rise to a slightly larger negative $\Delta T$ than methanol. The largest negative shifts observed were for tetraalkylammonium salts, which form large, weakly hydrated cations in water. A temperature shift $\Delta T$ of about $-5{ }^{\circ} \mathrm{C}$ was found for tetraethylammonium bromide $\left(\mathrm{Et}_{4} \mathrm{NBr}\right)$ while an even larger shift of ca. $-10{ }^{\circ} \mathrm{C}$ was measured for tetrabutylammonium bromide $\left(\mathrm{Bu}_{4}-\right.$ $\mathrm{NBr}$ ). Thus, certain cations are quite effective at stabilizing the isotropic phase and the reasons for this will be discussed below.

The negligible influence of anions on cromolyn phase behavior is underscored by Figure 5 , where we scale the $\Delta T$ data obtained with all the different sodium salts onto a master curve by plotting it against $\ln \left(1+v C_{\mathrm{s}} / C_{\mathrm{c}}\right)$. Here, $C_{\mathrm{c}}$ is the cromolyn concentration in molar units, $C_{\mathrm{s}}$ is the molar salt concentration, and $v$ is the valency of the anion. The $\Delta T$ data for Figure 5 are taken from Figures 2, 3, and 4, and represent a variety of anions, salt concentrations, and cromolyn concentrations. We note that the data for all $\mathrm{Na}^{+}$salts collapses onto a straight line in Figure 5. This means that salt effects can be empirically described by an equation of the form:

$$
\Delta T=A \ln \left(1+\frac{v C_{\mathrm{s}}}{C_{\mathrm{c}}}\right)=A\left\{\ln \left(C_{\mathrm{c}}+v C_{\mathrm{s}}\right)-\ln C_{\mathrm{c}}\right\}
$$

Note that $v C_{\mathrm{s}} / C_{\mathrm{c}}$ is the relative concentration of cations to cromolyn molecules for any given salt. The parameter $A$ in the above equation is the slope of the straight line in Figure 5. This slope is a measure of the extent to which the phase boundary will be shifted by a given concentration of a cation (for $\mathrm{Na}^{+}$, its value is $14.2 \mathrm{~K}$ ). The fact that the slope is steeper for $\mathrm{Na}^{+}$ salts compared to $\mathrm{Li}^{+}$salts indicates that $\mathrm{Na}^{+}$salts have a larger effect on the phase behavior.

Structure of Cromolyn-Salt Solutions: SANS. Having detailed the salt-induced changes in cromolyn phase behavior, we now describe the effects of salt on the structure of cromolyn solutions using SANS and cryo-TEM. For SANS experiments, solutions were made in $\mathrm{D}_{2} \mathrm{O}$ rather than $\mathrm{H}_{2} \mathrm{O}$. Salt effects on cromolyn in $\mathrm{D}_{2} \mathrm{O}$ were found to be very similar to those in $\mathrm{H}_{2} \mathrm{O}$. The only notable difference was that all coexistence curves were shifted upward by about $5{ }^{\circ} \mathrm{C}$ in $\mathrm{D}_{2} \mathrm{O}$ compared to $\mathrm{H}_{2} \mathrm{O}$.

Figure 6 shows SANS spectra for samples as a function of salt concentration and temperature. In Figure 6a, the effect of added salt is studied at a cromolyn concentration of $10 \mathrm{wt} \%$ and at a temperature well within the nematic phase $\left(10^{\circ} \mathrm{C}\right)$. The SANS spectrum for the salt-free sample shows a sharp, pronounced peak at high $q$. This is evidently a peak in the structure factor $S(q)$, arising because of strong positional correlations between the aggregates constituting the cromolyn nematic phase. The average distance between adjacent cromolyn aggregates can be calculated from the peak position $q_{0}$ by using 


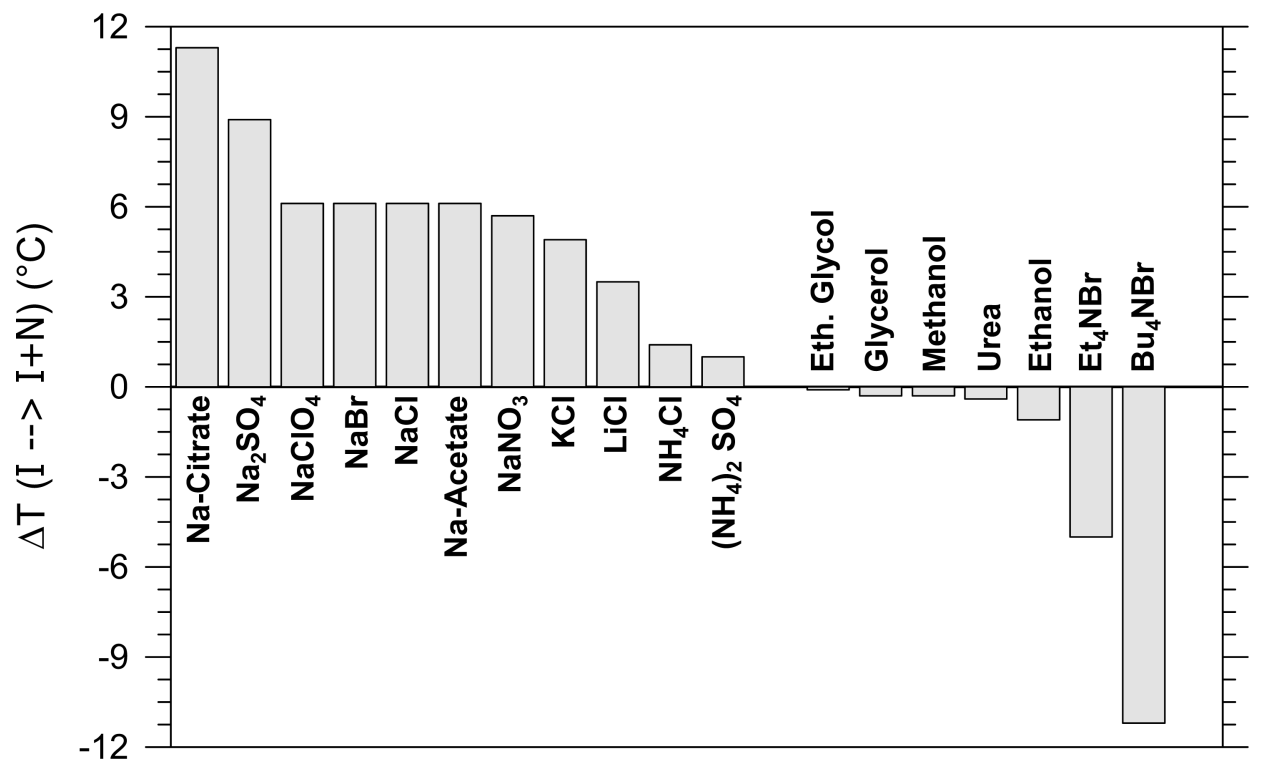

Figure 4. Shift in the $\mathrm{I} \rightarrow \mathrm{I}+\mathrm{N}$ transition temperature caused by the addition of $100 \mathrm{mM}$ of various additives to 9.5 wt $\%$ cromolyn solutions.

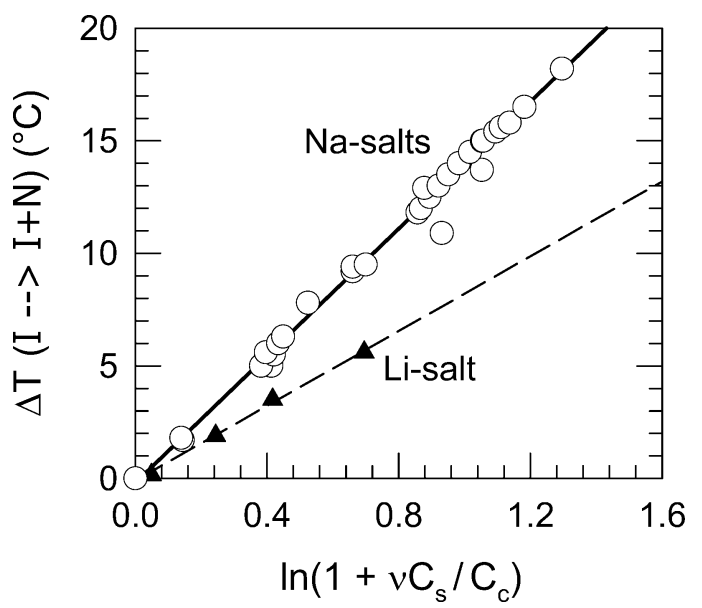

Figure 5. Scaled data for the shift in the $\mathrm{I} \rightarrow \mathrm{I}+\mathrm{N}$ phase transition temperature observed with various sodium and lithium salts. Data from Figures 2, 3, and 4 are replotted versus a scaled variable (see text for details).

the Bragg relation $d=2 \pi / q_{0}$. The value of $d$ is about $6 \mathrm{~nm}$ at this cromolyn concentration. When $\mathrm{NaCl}$ salt is added, the low- $q$ plateau in the intensity is progressively shifted upward, while the height of the peak decreases (the peak position itself does not change with salt concentration). A decrease in peak height suggests a weakening of interaggregate correlations, i.e., the average distance between aggregates is less well-defined at high salt concentration. Also, the low- $q$ plateau of the structure factor, $S(0)$, is a measure of the osmotic compressibility of the sample. ${ }^{18}$ So the data suggest an increase in effective osmotic compressibility, which translates into weaker repulsions between the aggregates, upon addition of salt.

Figure $6 \mathrm{~b}$ shows SANS spectra for the salt-free cromolyn sample in $\mathrm{D}_{2} \mathrm{O}$ as a function of temperature. This sample is nematic below $21^{\circ} \mathrm{C}$, two phase $(\mathrm{I}+\mathrm{N})$ between 21 and $25^{\circ} \mathrm{C}$, and isotropic above $25^{\circ} \mathrm{C}$. At $10^{\circ} \mathrm{C}$, the sample is well within the nematic phase and shows a strong peak, as discussed above. At $20^{\circ} \mathrm{C}$, the sample is still within the nematic phase but close to the phase boundary, and its SANS spectrum shows a lower peak. Note the similarities between the $20^{\circ} \mathrm{C}$ data in Figure $6 \mathrm{~b}$ and the $100 \mathrm{mM}$ salt data in Figure 6a. As before, we interpret the peak lowering to occur because the interaggregate spacing is less defined at higher temperatures. This could be because
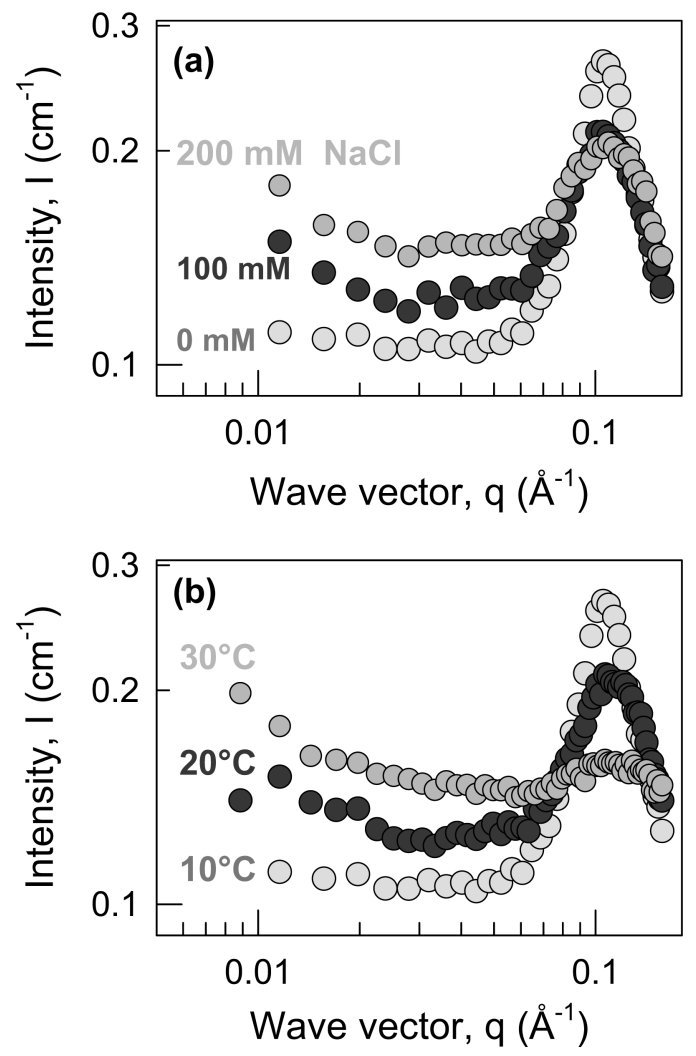

Figure 6. SANS data for $10 \mathrm{wt} \%$ cromolyn solutions in $\mathrm{D}_{2} \mathrm{O}$ with and without salt: (a) data as a function of salt $(\mathrm{NaCl})$ concentration at $10{ }^{\circ} \mathrm{C}$ and (b) data as a function of temperature for the sample with no salt.

the aggregates will have more translational energy at higher temperatures, so that they will tend to be more randomized and less ordered. At $30^{\circ} \mathrm{C}$, when the sample is in the isotropic phase, the peak in the SANS data is very weak, indicating a random structure with no order.

Structure of Cromolyn-Salt Solutions: Cryo-TEM. Structural differences between the salt-free and salt-containing cromolyn samples are further clarified by cryo-TEM. Figure 7 is a cryo-TEM image of a $10 \mathrm{wt} \%$ cromolyn sample in its nematic phase. The image shows a dense collection of parallel rods. The beginning and end of a single rod are not obvious, so 
$10 \%$ Cromolyn

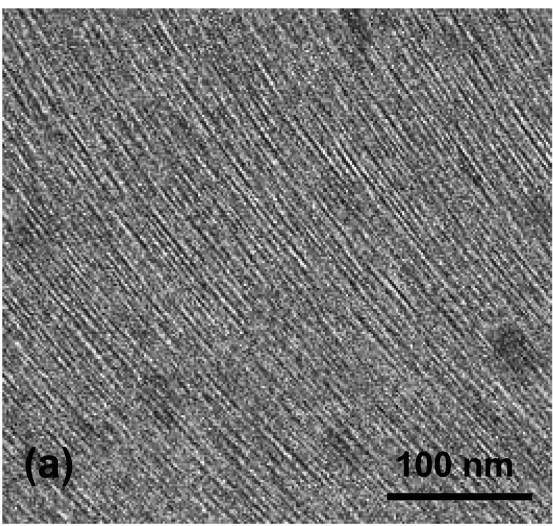

$10 \%$ Cromolyn $+350 \mathrm{mM} \mathrm{NaCl}$
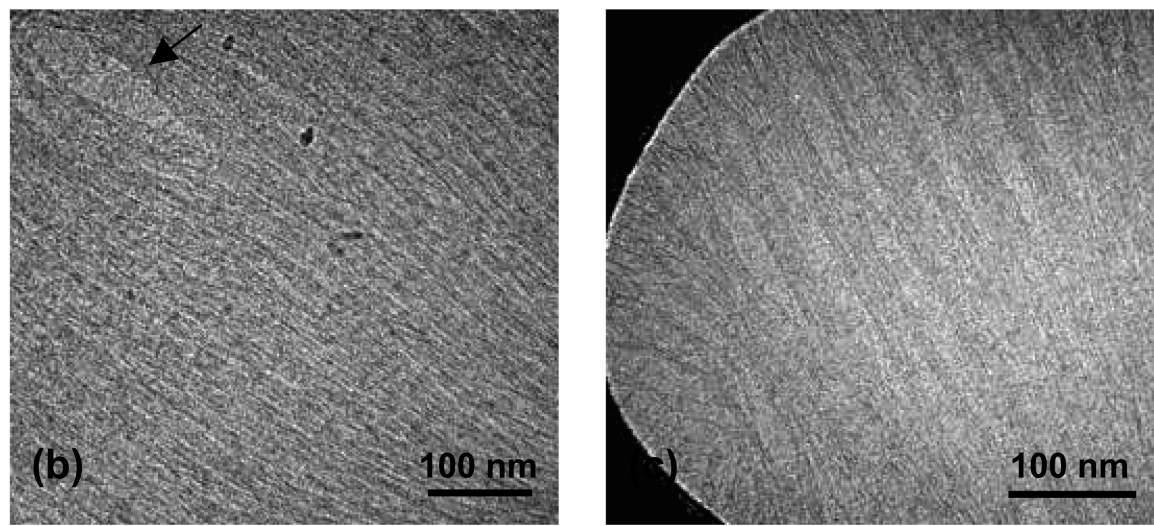

Figure 7. Cryo-TEM data for nematic cromolyn solutions with and without added salt: (a) $10 \mathrm{wt} \%$ cromolyn sample and (b, c) same cromolyn concentration and with $350 \mathrm{mM}$ of added $\mathrm{NaCl}$.

the order appears to be orientational, not positional. Moreover, the spacing between adjacent rods appears to be very welldefined and approximately a few nanometers, which is consistent with the Bragg spacing in SANS. Similar cryo-TEM images have been reported previously for other chromonic liquid crystals. ${ }^{16,19}$ The cryo-TEM images for samples with salt (Figure $7 \mathrm{~b}, \mathrm{c})$ show a similar arrangement of parallel rods for the most part; however, we also found some evidence for rod bundling as well. The onset of bundling is indicated by an arrow in Figure $7 \mathrm{~b}$, while Figure $7 \mathrm{c}$ reveals a number of such bundles. We can speculate that the bundling is a higher order aggregation of rods caused by the salt (i.e., the rods become sticky in the transverse direction). A similar bundling was also reported by using cryoTEM for a cyanine dye in the presence of salt. ${ }^{16}$ However, it is not clear if bundling is actually an equilibrium phenomenon. Note that the formation of bundles could explain why the interaggregate spacing from SANS becomes less defined in the presence of salt.

Rheology of Cromolyn-Salt Solutions. We have seen that salts exert a profound effect on the phase behavior and the microstructure of cromolyn solutions. It is interesting to examine if these effects are reflected in the sample rheology. We have therefore studied the rheology of cromolyn solutions with and without salt. Nematic liquid crystals tend to show a complex rheology, both in steady and oscillatory shear, ${ }^{20}$ and chromonic systems are no exception. ${ }^{21,22} \mathrm{~A}$ detailed discussion of cromolyn rheology will be reported elsewhere. Here we focus solely on salt effects under steady shear.

Typical steady-shear rheograms (viscosity as a function of shear-rate) are shown in Figure 8 for three samples containing $10 \mathrm{wt} \%$ cromolyn. The first sample has no salt, while the other two have 100 and $300 \mathrm{mM}$ of $\mathrm{NaCl}$, respectively. The data were collected at $10{ }^{\circ} \mathrm{C}$, which is within the nematic phase of all three samples. Three distinct regions are apparent in the plot: first a shear-thinning region (I), followed by a "Newtonian" region (II) where the viscosity is almost constant, and finally, a second shear-thinning region (III). (We should point out that Region I is sensitive to the experimental conditions and generally less reproducible.) Interestingly, a similar three-staged response is also observed for polymer nematics. ${ }^{20}$ For the purpose of this paper, the key finding from Figure 8 is that $\mathrm{NaCl}$ increases the viscosity over all three regions of the plot. Also, the higher the $\mathrm{NaCl}$ concentration, the higher the extent of viscosity increase. Incidentally, higher viscosities in the presence of salt are observed not only in the nematic phase, but also in the isotropic phase.

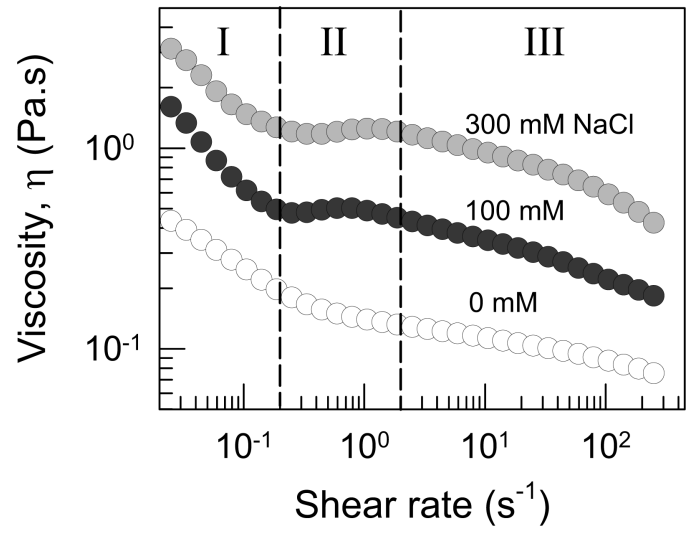

Figure 8. Steady-shear rheological data for nematic cromolyn samples with and without salt $(\mathrm{NaCl})$. The samples contain $10 \mathrm{wt} \%$ cromolyn and are nematic at the temperature studied $\left(10^{\circ} \mathrm{C}\right)$. Three distinct regimes are seen in the data as a function of increasing shear rate.

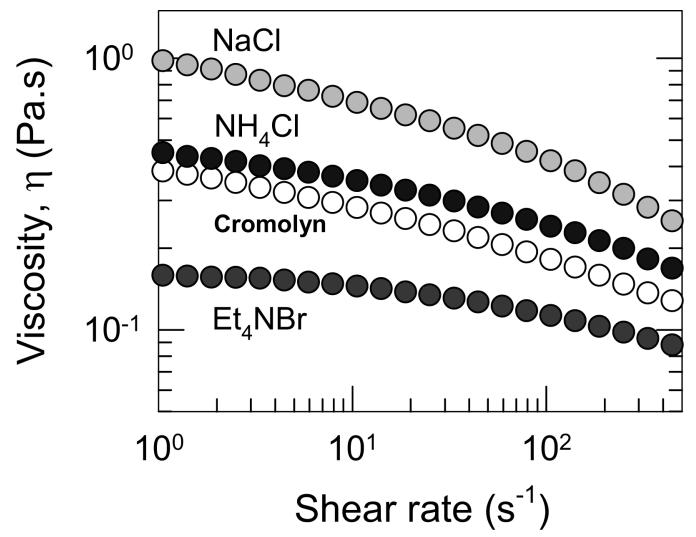

Figure 9. Steady-shear rheological data for nematic cromolyn samples with different salts. Data are shown only for Regions II and III from Figure 8. The cromolyn concentration is $10 \mathrm{wt} \%$ and the salt concentration is $100 \mathrm{mM}$ in all the samples. The data are collected at $5{ }^{\circ} \mathrm{C}$ where all the above samples are nematic.

Figure 9 compares the effect of different salts on the steadyshear rheological response at $5{ }^{\circ} \mathrm{C}$. Data are shown for pure cromolyn and for $100 \mathrm{mM} \mathrm{NaCl}, \mathrm{NH}_{4} \mathrm{Cl}$, and $\mathrm{Et}_{4} \mathrm{NBr}$. Recall from Figure 4 that $100 \mathrm{mM} \mathrm{NaCl}$ exerts a large upward shift on the phase boundary $\left(\Delta T \approx 6{ }^{\circ} \mathrm{C}\right)$, while $100 \mathrm{mM} \mathrm{NH}_{4} \mathrm{Cl}$ induces a smaller upward shift $\left(\Delta T \approx 2{ }^{\circ} \mathrm{C}\right)$, and $100 \mathrm{mM} \mathrm{Et}_{4^{-}}$ $\mathrm{NBr}$ induces an appreciable downward shift $\left(\Delta T \approx-5{ }^{\circ} \mathrm{C}\right)$. The viscosity data correlate nicely with the phase behavior results. Adding $100 \mathrm{mM} \mathrm{NaCl}$ causes the viscosity to double, 


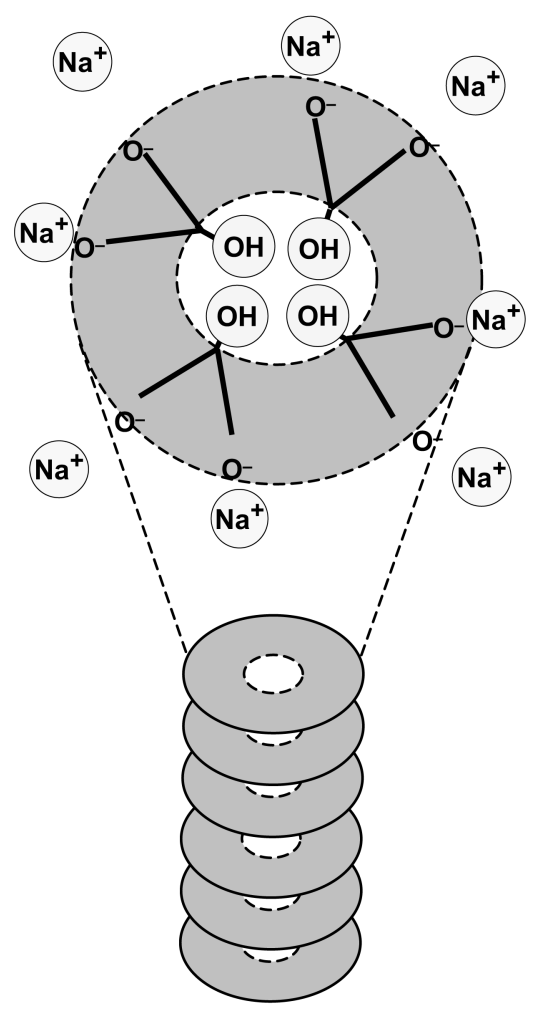

(a)

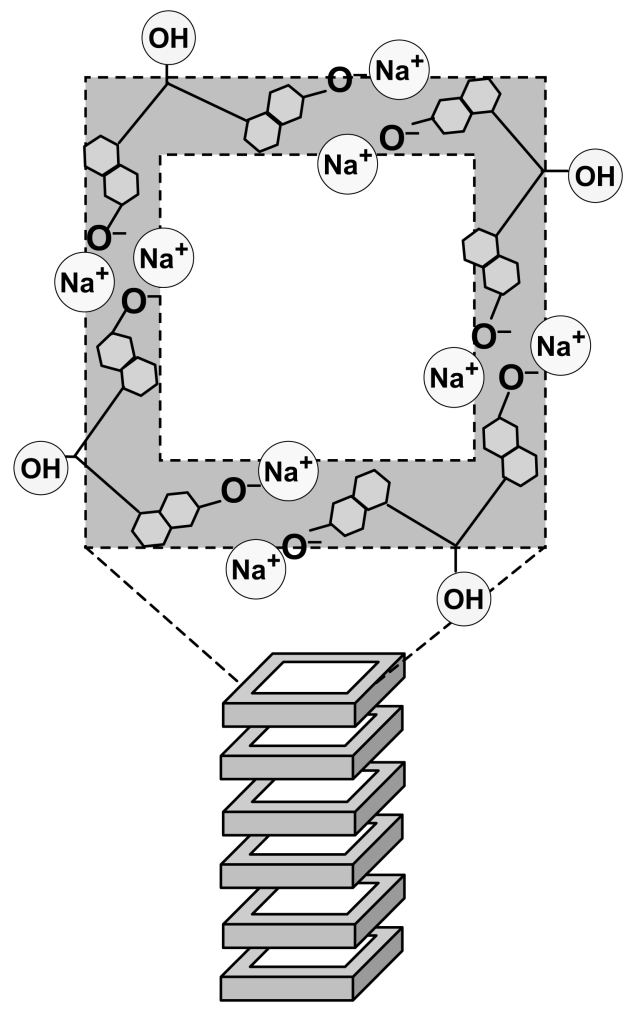

(b)

Figure 10. Schematic models for the cross-section of cromolyn aggregates: (a) Micelle-like model advanced by Labes et al..$^{25}$ and (b) square, hollow chimney model advanced by Lydon et al. ${ }^{7}$ In both cases, water is believed to be present in the interior of the aggregate.

while $100 \mathrm{mM} \mathrm{NH}_{4} \mathrm{Cl}$ induces a small increase in the viscosity. Finally, adding $100 \mathrm{mM} \mathrm{Et}_{4} \mathrm{NBr}$ lowers the viscosity to about half that of the cromolyn solution. The results therefore imply that an upward shift in the phase boundary correlates with an increase in the viscosity, and the larger the shift, the higher the viscosity. On the other hand, a lowering of the phase boundary correlates with a decrease in the viscosity.

\section{Discussion}

Our studies on salt effects in cromolyn solutions have uncovered some general patterns. Certain salts, particularly those with $\mathrm{Na}^{+}$or $\mathrm{K}^{+}$cations, have a dramatic effect on the phase behavior, stabilizing the nematic phase at the expense of the isotropic one. At the same time, these cations enhance the viscosity of the nematic solutions. Both the viscosity and the transition temperature rise monotonically with salt concentration in these cases. A plausible explanation is that these salts promote the growth of rodlike cromolyn aggregates. In other words, adding salt has a similar effect on rod growth as decreasing temperature. The nematic phase is formed when the rods reach a critical length and volume fraction. This condition is reached at a higher temperature in the presence of salt, which explains the upward shift in the phase boundary. At the same time, the presence of longer or more rods in solution at a given temperature can explain the higher viscosity.

While salt effects appear to follow a well-defined pattern, their specificity to the various counterions is unusual. This is perhaps the most significant finding from this study and our challenge is to find a mechanism that can explain all the results. In terms of a mechanism, we can first of all rule out a simple DLVO-based hypothesis whereby salt effects are treated purely based on ionic strength and counterion valency. According to DLVO theory, ${ }^{23}$ adding salt serves only to shrink the ionic double layer around individual ions on a cromolyn rod, thereby promoting aggregation. However, this cannot account for the rich diversity of specific effects reported in Figure 4 (e.g., for different univalent salts at the same concentration).

Next, we consider the possibility that our results reflect the Hofmeister series, ${ }^{24}$ i.e., how different salts influence the structure of water. However, there are several discrepancies with such a mechanism. First of all, we observe no effect whatsoever of the anion used (Figure 4). A partial Hofmeister series ${ }^{24}$ for anions reads as citrate $>\mathrm{SO}_{4}{ }^{2-}>$ acetate $>\mathrm{Cl}^{-}>\mathrm{NO}_{3}{ }^{-}>$ $\mathrm{ClO}_{4}{ }^{-}>\mathrm{SCN}^{-}$, with citrate and $\mathrm{SO}_{4}{ }^{2-}$ being salting-out anions whereas $\mathrm{ClO}_{4}^{-}$and $\mathrm{SCN}^{-}$are salting-in anions. We have deliberately studied anions over the entire series, but our results show that the phase behavior is insensitive to the anion and sensitive only to the cation concentration. Turning to the cations, their Hofmeister series reads as ${ }^{24} \mathrm{~N}\left(\mathrm{CH}_{3}\right)_{4}{ }^{+}>\mathrm{NH}_{4}{ }^{+}>\mathrm{Na}^{+}>$ $\mathrm{Li}^{+}>\mathrm{Mg}^{2+}>\mathrm{Ca}^{2+}>$ guanidinium. Our results are not entirely consistent with the above series either. For example, both $\mathrm{NH}_{4}{ }^{+}$ and $\mathrm{Li}^{+}$induce a smaller $\Delta T$ than $\mathrm{Na}^{+}$(Figure 4). Thus, our results do not support a mechanism based purely on how ions affect the structure of water.

We will now describe a plausible mechanism that can explain our data. The key assumption will be that cations from the added salt are effectively integrated into the structure of the cromolyn rods and participate in the self-assembly of these aggregates. In this context, it is pertinent to consider the arrangement of cromolyn molecules along the cross-section of a rod. Two existing hypotheses are shown in Figure 10. The structure in Figure 10a, with cromolyn molecules arranged as in a micelle, was put forward by Labes et al. ${ }^{25,26}$ In this model, the carboxylate groups point outward into the water, with the $\mathrm{Na}^{+}$ counterions possibly being released into the water. The $-\mathrm{OH}$ groups are clustered in the center of the rod and Labes et al. 
showed that "internal" water was also present at the center, possibly hydrogen bonded to the $-\mathrm{OH}$ groups. The alternative model, shown in Figure 10b, is due to Lydon et al. ${ }^{7,10}$ Here, the cromolyn rod is visualized as a hollow structure, with the cromolyn molecules arranged around its periphery and the $-\mathrm{OH}$ groups pointing outward. This model ascribes a more important role to the $\mathrm{Na}^{+}$counterions, which are visualized to form salt bridges between adjacent cromolyns and thereby hold the aggregate together.

Other models for cromolyn aggregates, termed "herringbone", "brickwork", "ladder", and "staircase" structures, have also been proposed. ${ }^{2,10}$ Our intention is not to discriminate between the above models. However, we believe that our results underscore the prominent role of counterions in aggregate formation, and we suggest that salt effects can be understood only by considering the binding of counterions (cations) to cromolyn aggregates. Such binding of cations has been suggested earlier by NMR studies. ${ }^{14}$ Note that we use the term binding in a general sense; this can occur either by adsorption (on the aggregate) and/or intercalation (into the aggregate).

The consequences of cation binding are postulated to be as follows. First, consider the binding of cations such as $\mathrm{Na}^{+}$. We suggest that the presence of additional $\mathrm{Na}^{+}$ions within the cromolyn rod promotes the growth of these aggregates. It is possible that the role of these cations is linked to salt bridging. Indeed, cations may not only serve as bridges between adjacent molecules in the plane of the cross-section, but could also serve as a link between molecules above and below this plane. In either case, the net effect will be to increase the length and/or number density of cromolyn rods in solution. This can explain the shift in phase boundary and the viscosity increase.

The binding hypothesis can also explain why it is the ratio of salt to cromolyn that controls the phase behavior. As shown by eq 1 and Figure 5, there is a universality to the transition temperature shifts observed for various $\mathrm{Na}^{+}$salts with different anions. To understand this, consider the equilibrium between cromolyn aggregates with and without counterions. At equilibrium, the difference in the chemical potentials of cromolyn aggregates with and without counterions, $\Delta \mu_{\mathrm{c}}$, is equal to zero. According to the thermodynamics of partial molar properties, ${ }^{27}$

$$
\Delta \mu_{\mathrm{c}}=\Delta \hat{H}_{\mathrm{c}}-T \Delta \hat{S}_{\mathrm{c}}
$$

where $\Delta \hat{H}_{\mathrm{c}}$ is the partial molar enthalpy difference and $\Delta \hat{S}_{\mathrm{c}}$ is the partial molar entropy difference. If we assume that the enhancement in aggregate stability is enthalpy-driven, $\Delta \hat{S}_{\mathrm{c}}$ can be represented as the partial molar entropy difference for ideal mixing of cromolyn aggregates with and without counterions: ${ }^{27}$

$$
\frac{\Delta \hat{S}_{\mathrm{c}}}{k_{B}}=-\left\{\ln \left(C_{\mathrm{c}}+\nu C_{\mathrm{s}}\right)-\ln C_{\mathrm{c}}\right\}=-\ln \left(1+\frac{\nu C_{\mathrm{s}}}{C_{\mathrm{c}}}\right)
$$

As defined earlier, $v$ is the valency of the anion and $v C_{\mathrm{s}}$ is the molar concentration of cations (counterions). Equation 3 thus shows that the partial molar entropy of cromolyn aggregates decreases due to counterion binding as the number of available microscopic states decreases. Therefore, the isotropic phase becomes less stable in the presence of bound counterions. At equilibrium, $\Delta \mu_{\mathrm{c}}=0$, which means that the loss of entropy should be compensated by the enthalpy change (release of heat). Combining eqs 2 and 3 :

$$
\frac{\Delta \hat{H}_{\mathrm{c}}}{k_{\mathrm{B}} T}=\frac{\Delta \hat{S}_{\mathrm{c}}}{k_{\mathrm{B}}}=-\ln \left(1+\frac{\nu C_{\mathrm{s}}}{C_{\mathrm{c}}}\right)
$$

Equation 4 shows that the sign of the partial molar enthalpy change is indeed negative, indicating a release of heat in the process of counterion binding (which means that binding is a favorable process). This heat release must be proportional to the temperature shift of the phase boundary $\Delta T$ (compare eqs 4 and 1). We thus see why $\Delta T$ is positive, i.e., why the phase boundary is shifted upward upon adding salt. This also gives a theoretical basis to the empirical form of eq 1 and for the scaled horizontal axis in Figure 5. Our analysis thus explains why $\Delta T$ is controlled by the ratio of counterion concentration $v C_{\mathrm{s}}$ to cromolyn concentration $C_{\mathrm{c}}$.

Cation binding can also explain the results we observe with different salts. We believe that these results are linked to cation size. $\mathrm{Na}^{+}$counterions are liable to be a perfect fit within a cromolyn rod, whereas cations that are either smaller or larger than $\mathrm{Na}^{+}$will not be as good a fit. This can explain why both cations that are smaller (e.g. $\mathrm{Li}^{+}$) or larger (e.g. $\mathrm{NH}_{4}^{+}$) than $\mathrm{Na}^{+}$do not promote rod growth to the same extent and thereby induce smaller $\Delta T$ values (Figure 4 ). On the other hand, $\mathrm{K}^{+}$ ions are similar in size to $\mathrm{Na}^{+}$and give about the same $\Delta T$. Finally, large cations such as $\mathrm{Et}_{4} \mathrm{~N}^{+}$and $\mathrm{Bu}_{4} \mathrm{~N}^{+}$possibly also bind to the rod, but they are such a poor fit that they actually suppress rod growth. This in turn leads to a stabilization of the isotropic phase at the expense of the nematic phase, i.e., a negative $\Delta T$, and the decrease in rod size also lowers the solution viscosity.

Cation binding can also explain other experimental findings. For example, the presence of bound cations may induce attractive interactions between molecules present on adjacent cromolyn rods. This may explain the bundle formation that we observe in some cryo-TEM images. Along the same vein, cation binding may also explain the precipitation observed with divalent cations such as $\mathrm{Mg}^{2+}$ and $\mathrm{Ca}^{2+}$. This could arise because bound divalent cations form bridges with cromolyn molecules on other aggregates, leading to a macroscopic precipitation. In summary, a mechanism involving cation binding appears to be consistent with the majority of our experimental results.

\section{Conclusions}

In this study, we have shown that the addition of salts can cause dramatic changes to the phase behavior, structure, and rheology of nematic cromolyn solutions. We hypothesize that salt effects are related to the binding of salt cations to the rodlike aggregates of cromolyn. The binding of $\mathrm{Na}^{+}$and $\mathrm{K}^{+}$cations promotes the growth and assembly of cromolyn rods, which in turn causes an upward shift $(\Delta T)$ in the phase boundaries and also increases the viscosity of cromolyn solutions. Other monovalent cations such as $\mathrm{Li}^{+}$and $\mathrm{NH}_{4}^{+}$induce smaller upward shifts, presumably because their molecular size is different from those of the $\mathrm{Na}^{+}$counterions. Finally, very large, hydrophobic cations such as tetraethylammonium and tetrabutylammonium, shift the phase boundary downward, i.e., they suppress the formation of the nematic phase. We hypothesize that these large cations disrupt the orderly packing of cromolyn molecules within a rod, thus decreasing the tendency of cromolyn to aggregate.

Acknowledgment. We would like to acknowledge NIST for facilitating the SANS experiments performed as part of this work. D.D. acknowledges the support of the Israel Science Foundation through equipment grant No. 9059/03.

\section{References and Notes}

(1) Attwood, T. K.; Lydon, J. E.; Hall, C.; Tiddy, G. J. T. Liq. Cryst. 1990, 7, 657 . 
(2) Lydon, J. E. Curr. Opin. Colloid Interface Sci. 1998, 3, 458.

(3) Lydon, J. E. Curr. Opin. Colloid Interface Sci. 2004, 8, 480. 349.

(4) Attwood, T. K.; Lydon, J. E. Mol. Cryst. Liq. Cryst. 1984, 108,

(5) Attwood, T. K.; Lydon, J. E.; Jones, F. Liq. Cryst. 1986, 1, 499. 23,343

(7) Lydon, J. E. Mol. Cryst. Liq. Cryst. 1980, 64, 19

(8) Lee, H.; Labes, M. M. Mol. Cryst. Liq. Cryst. 1983, 91, 53.

(9) Goldfarb, D.; Luz, Z.; Spielberg, N.; Zimmermann, H. Mol. Cryst. Liq. Cryst. 1985, 126, 225.

(10) Attwood, T. K.; Lydon, J. E. Mol. Cryst. Liq. Cryst. 1986, 4, 9.

(11) Nastishin, Y. A.; Liu, H.; Shiyanovskii, S. V.; Lavrentovich, O.

D.; Kostko, A. F.; Anisimov, M. A. Phys. Rev. E 2004, 70.

(12) Lee, H.; Labes, M. M. Mol. Cryst. Liq. Cryst. 1982, 84, 137.

(13) Yu, L. J.; Saupe, A. Mol. Cryst. Liq. Cryst. 1982, 80, 129.

(14) Perahia, D.; Goldfarb, D.; Luz, Z. Mol. Cryst. Liq. Cryst. 1984, $108,107$.

(15) Mundy, K.; Sleep, J. C.; Lydon, J. E. Liq. Cryst. 1995, 19, 107.

(16) von Berlepsch, H.; Bottcher, C. J. Phys. Chem. B 2002, 106, 3146.
(17) Kuzma, M.; Saupe, A. Mol. Cryst. Liq. Cryst. 1983, 90, 349.

(18) Zemb, T.; Lindner, P., Eds. Neutron, X-Ray and Light Scattering; Elsevier: Amsterdam, The Netherlands, 1991.

(19) von Berlepsch, H.; Bottcher, C.; Dahne, L. J. Phys. Chem. B 2000, $104,8792$.

(20) Larson, R. G. The Structure and Rheology of Complex Fluids; Oxford University Press: New York, 1998.

(21) Kuzma, M.; Hui, Y. W.; Labes, M. M. Mol. Cryst. Liq. Cryst. 1989, $172,211$.

(22) Golovanov, A. V.; Kaznacheev, A. V.; Sonin, A. S. Izv. Akad. Nauk Ser. Fiz. 1996, 60, 43.

(23) Evans, D. F.; Wennerstrom, H. The Colloidal Domain; WileyVCH: New York, 2001.

(24) Cacace, M. G.; Landau, E. M.; Ramsden, J. J. Q. Rev. Biophys. 1997, 30, 241.

(25) Hui, Y. W.; Labes, M. M. J. Phys. Chem. 1986, 90, 4064

(26) Ramesh, V.; Chien, H. S.; Labes, M. M. J. Phys. Chem. 1987, 91, 5937.

(27) Sandler, S. I. Chemical and Engineering Thermodynamics; Wiley: New York, 1999. 